\title{
Schwarz boundary value problem for the Cauchy-Riemann equation in a rectangle
}

Ying Wang ${ }^{1 *}$ (D) and Xuefang Zhao ${ }^{2}$

\author{
${ }^{*}$ Correspondence: \\ wangyingyezi@sina.com \\ 'School of Statistics and \\ Mathematics, Zhongnan University \\ of Economics and Law, Wuhan, \\ 430072, P.R. China \\ Full list of author information is \\ available at the end of the article
}

\begin{abstract}
In this paper, Schwarz problem for the inhomogeneous Cauchy-Riemann equation in a rectangle is investigated explicitly. By the parqueting reflection principle and the Cauchy-Pompeiu formula, a modified Schwarz-Poisson representation formula in a rectangle is constructed. In particular, the boundary behaviors for the related Schwarz-type and Pompeiu-type operators at the corner points are discussed in detail.
\end{abstract}

MSC: $30 \mathrm{E} 25 ; 35 \mathrm{C} 15 ; 35 \mathrm{~F} 45$

Keywords: Schwarz-Poisson formula; Cauchy-Riemann equation; Schwarz problem

\section{Introduction}

There are many investigations of boundary value problems for its theoretical significance and extensive applications in mathematical physics, such as elasticity theory, potential theory, medical imaging. Numerous results have been achieved for boundary value problems in different particular domains; see [1-10]. The basic boundary value problems are the Schwarz, Dirichlet, Robin and Neumann problems. In general, the well-known conformal invariance of the reflection across circles and lines can be used to find a Schwarz operator for simple domains like circles, half circles, rings, half planes, etc. But the explicit Schwarz-Poisson formula in a rectangle cannot be simply obtained from the classical Schwarz-Poisson formula on the unit disc or the half-plane by conformal mapping. In $[11,12]$, the authors introduced a method of plane parqueting, which can be used to determine a Schwarz operator, the harmonic Green and Neumann functions, and requires that reflecting the domain at all of these circular or straight curves produces a parqueting of whole complex plane with possible exception of singular points. In $[7,10,13]$, the authors discussed Schwarz and Dirichlet problems in an isosceles orthogonal triangle and an equilateral triangle via different series. Also the Green and Neumann functions for a strip and a rectangle were established in detail in [6]. In the present paper, we construct a modified Schwarz-Poisson representation formula by the parqueting reflection principle and Cauchy-Pompeiu formula in a rectangle, discussing the related Schwarz problem explicitly.

Let $\Omega$ be a domain in the complex plane $\mathbb{C}$ defined by

$$
\Omega=\{z=x+i y: 0<x<a, 0<y<b\}
$$

(c) 2016 Wang and Zhao. This article is distributed under the terms of the Creative Commons Attribution 4.0 International License (http://creativecommons.org/licenses/by/4.0/), which permits unrestricted use, distribution, and reproduction in any medium, provided you give appropriate credit to the original author(s) and the source, provide a link to the Creative Commons license, and indicate if changes were made. 
where $0, a, \mu=a+i b, v=i b$ are four corner points of the domain $\Omega$. The boundary $\partial \Omega=$ $[0, a] \cup[a, \mu] \cup[\mu, v] \cup[v, 0]$ is oriented counter-clockwise. That is, the oriented line $[0, a]$ is parameterized by $t \mapsto t, t \in[0, a]$, the segment $[a, \mu]$ is parameterized by $t \mapsto a+i t$, $t \in[0, b]$, the oriented segment $[\mu, v]$ is parameterized by $t \mapsto t+i b, t \in[a, 0]$, and the oriented segment $[v, 0]$ is parameterized by $t \mapsto i t, t \in[b, 0]$.

Lemma $1.1([14-16])$ If $w \in C^{1}(\Omega ; \mathbb{C}) \cap C(\bar{\Omega} ; \mathbb{C})$, then

$$
\frac{1}{2 \pi i} \int_{\partial \Omega} w(\zeta) \frac{\mathrm{d} \zeta}{\zeta-z}-\frac{1}{\pi} \int_{\Omega} w_{\bar{\zeta}}(\zeta) \frac{\mathrm{d} \xi \mathrm{d} \eta}{\zeta-z}= \begin{cases}w(z), & z \in \Omega \\ 0, & z \notin \bar{\Omega}\end{cases}
$$

and

$$
-\frac{1}{2 \pi i} \int_{\partial \Omega} w(\zeta) \frac{\mathrm{d} \bar{\zeta}}{\overline{\zeta-z}}-\frac{1}{\pi} \int_{\Omega} w_{\zeta}(\zeta) \frac{\mathrm{d} \xi \mathrm{d} \eta}{\overline{\zeta-z}}= \begin{cases}w(z), & z \in \Omega \\ 0, & z \notin \bar{\Omega}\end{cases}
$$

where $\Omega$ is the rectangle defined by (1) and $\zeta=\xi+i \eta, \xi, \eta \in \mathbb{R}$.

Reflecting the point $z \in \Omega$ at $[a, \mu]$, the symmetric point is $z_{1}=2 a-\bar{z}$ and the domain $\Omega$ is bijectively mapped onto a rectangle $\Omega_{1}=\{z=x+i y: a \leq x \leq 2 a, 0 \leq y \leq b\}$. Furthermore, the symmetric point of $z_{1}$ at $[\mu, v]$ is $z_{2}=-z+2 a+2 b i$ and the domain $\Omega_{1}$ is bijectively mapped onto a rectangle $\Omega_{2}=\{z=x+i y: a \leq x \leq 2 a, b \leq y \leq 2 b\}$. Continuing the reflection at $[a, \mu]$, the symmetric point of $z_{2}$ is $z_{3}=\bar{z}+2 b i$ and the domain $\Omega_{2}$ is bijectively mapped onto a rectangle $\Omega_{3}=\{z=x+i y: 0 \leq x \leq a, b \leq y \leq 2 b\}$. Finally, reflecting $z_{3}$ at $[\mu, v]$, the symmetric point of $z_{3}$ is just point $z$. Therefore, let $\Omega_{0,0}=\Omega \cup \Omega_{1} \cup \Omega_{2} \cup \Omega_{3}$, we know that $\Omega_{0,0}$ can be a basic rectangle and the reflection along the horizontal and vertical direction is equivalent to extending the basic rectangle $\Omega_{0,0}$ by double periods $2 b i, 2 a$, respectively (see Figure 1).

Denoting $\omega_{m n}=2(m a+n b i), m, n \in \mathbb{Z}$, and

$$
\Omega_{m, n}=\left\{z+\omega_{m n}: z \in \Omega_{0,0}, m, n \in \mathbb{Z}\right\}
$$

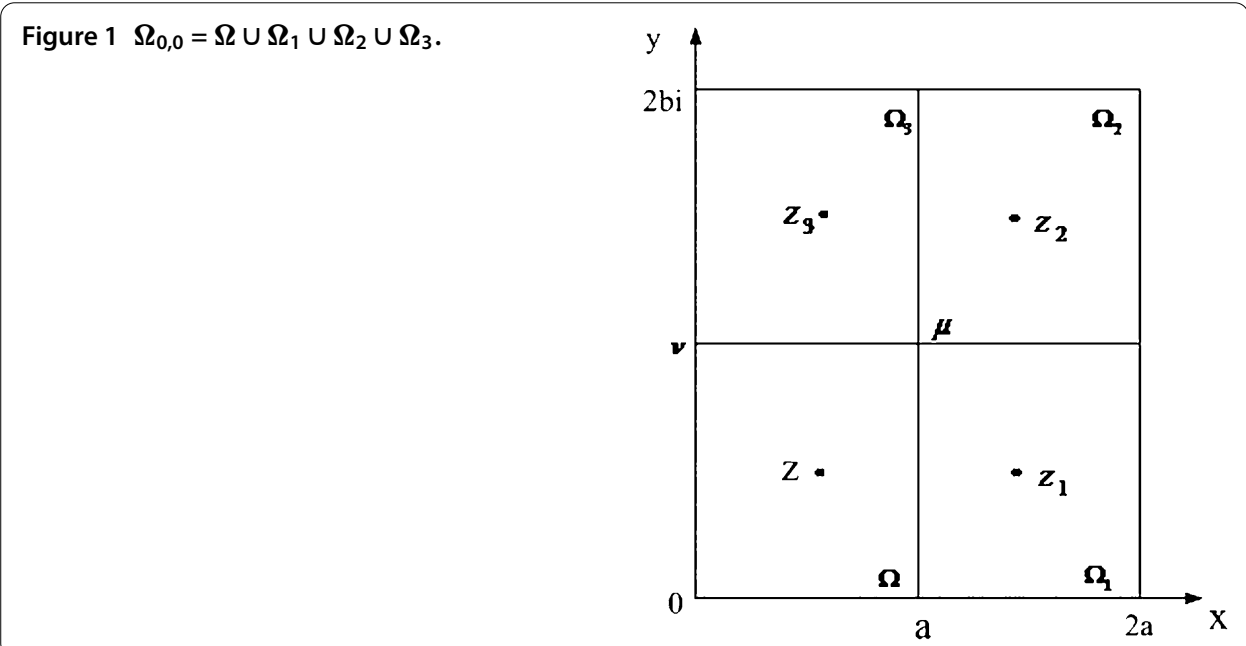


then $\mathbb{C}=\bigcup_{m, n=-\infty}^{+\infty} \bar{\Omega}_{m, n}$. By the technique of plane parqueting, the complex plane is divided into infinitely rectangles, which are congruent to the domain $\Omega$.

\section{Schwarz-Poisson formula}

Suppose $R_{M, N}=\{(k, j):|k| \leq M,|j| \leq N, k, j \in \mathbb{Z}\}$ for $M, N \in \mathbb{N}$ be a finite set of dou-

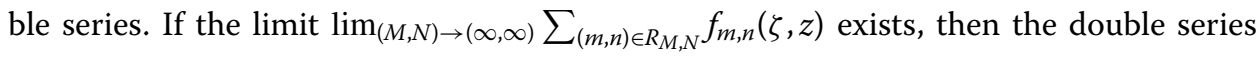
$\sum_{(m, n) \in \mathbb{Z} \times \mathbb{Z}} f_{m, n}(\zeta, z)$, simply $\sum_{m, n} f_{m, n}(\zeta, z)$ is convergent along the rectangle, that is,

$$
\sum_{m, n} f_{m, n}(\zeta, z)=\lim _{(M, N) \rightarrow(\infty, \infty)} \sum_{(m, n) \in R_{M, N}} f_{m, n}(\zeta, z)
$$

Similarly in $[10,17]$, the following result is true.

Lemma 2.1 Suppose that $S, E, W \subset \mathbb{C}$ be three bounded sets. If $S \cap E_{m, n}=\emptyset, S \cap W_{m, n}=\emptyset$ for all $(m, n) \in \mathbb{Z} \times \mathbb{Z}$, then the double series

$$
\sum_{m, n}\left[\frac{1}{\zeta-z-\omega_{m n}}-\frac{1}{\zeta-w-\omega_{m n}}\right]
$$

is uniformly convergent for $(\zeta, z, w) \in S \times E \times W$ with $E_{m, n}=\left\{z+\omega_{m n}: z \in E\right\}$ and $W_{m, n}=$ $\left\{z+\omega_{m n}: z \in W\right\}$.

Let

$$
\begin{aligned}
q_{m, n}(\zeta, z) & =\frac{1}{\zeta-z-\omega_{m n}}+\frac{1}{\zeta+z-\omega_{m n}} \\
& =\frac{1}{\zeta-z-2 m a-2 n b i}+\frac{1}{\zeta+z-2 m a-2 n b i},
\end{aligned}
$$

then we have the following result.

Theorem 2.1 Any $w \in C^{1}(\Omega ; \mathbb{C}) \cap C(\bar{\Omega} ; \mathbb{C})$ can be represented as

$$
w(z)=w(\alpha)+\frac{1}{2 \pi i} \int_{\partial \Omega} w(\zeta) \Xi_{\alpha}(\zeta, z) \mathrm{d} \zeta-\frac{1}{\pi} \int_{\Omega} w_{\bar{\zeta}}(\zeta) \Xi_{\alpha}(\zeta, z) \mathrm{d} \xi \mathrm{d} \eta, \quad z \in \Omega
$$

and, for $z \in \Omega$,

$$
\begin{aligned}
w(z)= & w(\alpha)+\frac{1}{\pi i} \int_{\partial \Omega} \operatorname{Re} w(\zeta) \Xi_{\alpha}(\zeta, z) \mathrm{d} \zeta \\
& -\frac{1}{\pi} \int_{\Omega}\left[w_{\bar{\zeta}}(\zeta) \Xi_{\alpha}(\zeta, z)-\overline{w_{\bar{\zeta}}(\zeta)} \Xi_{\alpha}(\bar{\zeta}, z)\right] \mathrm{d} \xi \mathrm{d} \eta
\end{aligned}
$$

where $\alpha$ is a fixed point in $\Omega$ and

$$
\Xi_{\alpha}(\zeta, z)=\sum_{m, n}\left[q_{m, n}(\zeta, z)-q_{m, n}(\zeta, \alpha)\right]
$$


Proof For $z \in \Omega$, we know $\pm \bar{z}+\omega_{m n},-z+\omega_{m n} \notin \Omega$ for $(m, n) \in \mathbb{Z} \times \mathbb{Z}$, and $z+\omega_{m n} \notin \Omega$ for $(m, n) \neq(0,0)$. Then by Lemma 1.1 , one has, for $z \in \Omega$,

$$
\begin{aligned}
& \frac{1}{2 \pi i} \int_{\partial \Omega} \frac{w(\zeta)}{\zeta-z} \mathrm{~d} \zeta-\frac{1}{\pi} \int_{\Omega} w_{\bar{\zeta}}(\zeta) \frac{\mathrm{d} \xi \mathrm{d} \eta}{\zeta-z}=w(z), \\
& \frac{1}{2 \pi i} \int_{\partial \Omega} \frac{w(\zeta)}{\zeta-z-\omega_{m n}} \mathrm{~d} \zeta-\frac{1}{\pi} \int_{\Omega} w_{\bar{\zeta}}(\zeta) \frac{\mathrm{d} \xi \mathrm{d} \eta}{\zeta-z-\omega_{m n}}=0, \quad(m, n) \neq(0,0), \\
& \frac{1}{2 \pi i} \int_{\partial \Omega} \frac{w(\zeta)}{\zeta+z-\omega_{m n}} \mathrm{~d} \zeta-\frac{1}{\pi} \int_{\Omega} w_{\bar{\zeta}}(\zeta) \frac{\mathrm{d} \xi \mathrm{d} \eta}{\zeta+z-\omega_{m n}}=0, \\
& \frac{1}{2 \pi i} \int_{\partial \Omega} \frac{w(\zeta)}{\zeta-\bar{z}-\omega_{m n}} \mathrm{~d} \zeta-\frac{1}{\pi} \int_{\Omega} w_{\bar{\zeta}}(\zeta) \frac{\mathrm{d} \xi \mathrm{d} \eta}{\zeta-\bar{z}-\omega_{m n}}=0, \\
& \frac{1}{2 \pi i} \int_{\partial \Omega} \frac{w(\zeta)}{\zeta+\bar{z}-\omega_{m n}} \mathrm{~d} \zeta-\frac{1}{\pi} \int_{\Omega} w_{\bar{\zeta}}(\zeta) \frac{\mathrm{d} \xi \mathrm{d} \eta}{\zeta+\bar{z}-\omega_{m n}}=0 .
\end{aligned}
$$

Adding (7), (8) and (9), and taking the sum for all the indices $(m, n) \in R_{M, N}$, we obtain

$$
w(z)=\frac{1}{2 \pi i} \int_{\partial \Omega} w(\zeta) Q_{M, N}(\zeta, z) \mathrm{d} \zeta-\frac{1}{\pi} \int_{\Omega} w_{\bar{\zeta}}(\zeta) Q_{M, N}(\zeta, z) \mathrm{d} \xi \mathrm{d} \eta
$$

which implies that

$$
w(\alpha)=\frac{1}{2 \pi i} \int_{\partial \Omega} w(\zeta) Q_{M, N}(\zeta, \alpha) \mathrm{d} \zeta-\frac{1}{\pi} \int_{\Omega} w_{\bar{\zeta}}(\zeta) Q_{M, N}(\zeta, \alpha) \mathrm{d} \xi \mathrm{d} \eta,
$$

where

$$
Q_{M, N}(\zeta, z)=\sum_{(m, n) \in R_{M, N}} q_{m, n}(\zeta, z)
$$

Subtracting (13) from (12), then

$$
\begin{aligned}
w(z)= & w(\alpha)+\frac{1}{2 \pi i} \int_{\partial \Omega} w(\zeta)\left[Q_{M, N}(\zeta, z)-Q_{M, N}(\zeta, \alpha)\right] \mathrm{d} \zeta \\
& -\frac{1}{\pi} \int_{\Omega} w_{\bar{\zeta}}(\zeta)\left[Q_{M, N}(\zeta, z)-Q_{M, N}(\zeta, \alpha)\right] \mathrm{d} \xi \mathrm{d} \eta .
\end{aligned}
$$

Putting $(M, N) \rightarrow(\infty, \infty)$ and from Lemma 2.1, we get (5).

Let

$$
g_{m, n}(\zeta, \bar{z})= \begin{cases}q_{m,-n}(\zeta, \bar{z}), & \zeta \in[0, a], \\ q_{-m+1, n}(\zeta, \bar{z}), & \zeta \in[a, \mu], \\ q_{m,-n+1}(\zeta, \bar{z}), & \zeta \in[\mu, \nu], \\ q_{-m, n}(\zeta, \bar{z}), & \zeta \in[\nu, 0],\end{cases}
$$

then from the relationship

$$
\zeta= \begin{cases}\bar{\zeta}, & \zeta \in[0, a] \\ 2 a-\bar{\zeta}, & \zeta \in[a, \mu] \\ \bar{\zeta}+2 b i, & \zeta \in[\mu, v] \\ -\bar{\zeta}, & \zeta \in[v, 0]\end{cases}
$$


we obtain

$$
\overline{q_{m, n}(\zeta, z)} \mathrm{d} \bar{\zeta}=g_{m, n}(\zeta, \bar{z}) \mathrm{d} \zeta, \quad \zeta \in \partial \Omega
$$

Furthermore, by (18) and taking the sum of conjugations of (10) and (11) for all the indices $(m, n) \in R_{M, N}$, we have

$$
\frac{1}{2 \pi i} \int_{\partial \Omega} \overline{w(\zeta)} H_{M, N}(\zeta, z) \mathrm{d} \zeta+\frac{1}{\pi} \int_{\Omega} \overline{w_{\bar{\zeta}}(\zeta)} H_{M, N}(\bar{\zeta}, z) \mathrm{d} \xi \mathrm{d} \eta=0
$$

where

$$
H_{M, N}(\zeta, z)= \begin{cases}\sum_{(m, n) \in R_{M, N}} q_{m,-n}(\zeta, z), & \zeta \in[0, a], \\ \sum_{(m, n) \in R_{M, N}} q_{-m+1, n}(\zeta, z), & \zeta \in[a, \mu], \\ \sum_{(m, n) \in R_{M, N}} q_{m,-n+1}(\zeta, z), & \zeta \in[\mu, \nu], \\ \sum_{(m, n) \in R_{M, N}} q_{-m, n}(\zeta, z), & \zeta \in[\nu, 0], \\ \sum_{(m, n) \in R_{M, N}} q_{m,-n}(\zeta, z), & \zeta \in \Omega .\end{cases}
$$

Obviously,

$$
\frac{1}{2 \pi i} \int_{\partial \Omega} \overline{w(\zeta)} H_{M, N}(\zeta, \alpha) \mathrm{d} \zeta+\frac{1}{\pi} \int_{\Omega} \overline{w_{\bar{\zeta}}(\zeta)} H_{M, N}(\bar{\zeta}, \alpha) \mathrm{d} \xi \mathrm{d} \eta=0
$$

Subtracting (21) from the sum of (19) and (15), we know

$$
\begin{aligned}
w(z)= & w(\alpha)+\frac{1}{2 \pi i} \int_{\partial \Omega}[w(\zeta) A(\zeta, z)+\overline{w(\zeta)} B(\zeta, z)] \mathrm{d} \zeta \\
& -\frac{1}{\pi} \int_{\Omega}\left[w_{\bar{\zeta}}(\zeta) A(\zeta, z)-\overline{w_{\bar{\zeta}}(\zeta)} B(\bar{\zeta}, z)\right] \mathrm{d} \xi \mathrm{d} \eta
\end{aligned}
$$

with

$$
A(\zeta, z)=Q_{M, N}(\zeta, z)-Q_{M, N}(\zeta, \alpha)
$$

and

$$
B(\zeta, z)=H_{M, N}(\zeta, z)-H_{M, N}(\zeta, \alpha)
$$

From Lemma 2.1, (14) and (20), we obtain, for $z \in \Omega$,

$$
\lim _{(M, N) \rightarrow(\infty, \infty)} A(\zeta, z)=\lim _{(M, N) \rightarrow(\infty, \infty)} B(\zeta, z)=\sum_{m, n}\left[q_{m, n}(\zeta, z)-q_{m, n}(\zeta, \alpha)\right]
$$

and

$$
\lim _{(M, N) \rightarrow(\infty, \infty)} B(\bar{\zeta}, z)=\sum_{m, n}\left[q_{m, n}(\bar{\zeta}, z)-q_{m, n}(\bar{\zeta}, \alpha)\right]
$$

Letting $(M, N) \rightarrow(\infty, \infty)$ for (22), (6) is obviously true. 


\section{Schwarz problem}

The classical Schwarz kernel for the upper half-plane $\mathbb{C}^{+}$is

$$
\frac{z-\bar{z}}{|x-z|^{2}}, \quad(x, z) \in(-\infty,+\infty) \times \mathbb{C}^{+}
$$

and satisfies (see [14])

$$
\begin{aligned}
& \lim _{z \in \mathbb{C}^{+}, z \rightarrow t} \frac{1}{2 \pi i} \int_{c}^{d} \rho(x)\left[\frac{1}{x-z}-\frac{1}{x-\bar{z}}\right] \mathrm{d} x \\
& =\rho(t), \quad t \in(c, d) \subset(-\infty,+\infty),
\end{aligned}
$$

with $\rho \in C([c, d], \mathbb{C}), c<d$.

Lemma 3.1 For $\rho \in C(\partial \Omega, \mathbb{C}), z \in \Omega$, we obtain

$$
\begin{aligned}
& \lim _{z \rightarrow t} \frac{1}{2 \pi i} \int_{[a, \mu]} \rho(\xi)\left[\frac{1}{\xi-z}-\frac{1}{\xi+\bar{z}-2 a}\right] \mathrm{d} \xi=\rho(t), \quad t \in(a, \mu), \\
& \lim _{z \rightarrow t} \frac{1}{2 \pi i} \int_{[\mu, \nu]} \rho(\xi)\left[\frac{1}{\xi-z}-\frac{1}{\xi-\bar{z}-2 b i}\right] \mathrm{d} \xi=\rho(t), \quad t \in(\mu, v), \\
& \lim _{z \rightarrow t} \frac{1}{2 \pi i} \int_{[\nu, 0]} \rho(\xi)\left[\frac{1}{\xi-z}-\frac{1}{\xi+\bar{z}}\right] \mathrm{d} \xi=\rho(t), \quad t \in(\nu, 0) .
\end{aligned}
$$

Proof When $\xi \in[a, \mu]$ and letting $\xi=a+i y$, then $y \in[0, b]$ and

$$
\begin{aligned}
& \lim _{z \rightarrow t, t \in(a, \mu)} \frac{1}{2 \pi i} \int_{[a, \mu]} \rho(\xi)\left[\frac{1}{\xi-z}-\frac{1}{\xi+\bar{z}-2 a}\right] \mathrm{d} \xi \\
& \quad=\lim _{z \rightarrow t, t \in(a, \mu)} \frac{1}{2 \pi i} \int_{0}^{b} \rho(a+i y)\left[\frac{1}{y-(a-z) i}-\frac{1}{y-(\bar{z}-a) i}\right] \mathrm{d} y .
\end{aligned}
$$

For $z \in \Omega$ and $z \rightarrow t \in(a, \mu)$, we obtain $(a-z) i \in \mathbb{C}^{+}$and $\rightarrow(a-t) i \in(0, b)$. Thus, from the classical Schwarz kernel theory (23), the first limit in Lemma 3.1 equals $\rho(t)$.

For $\xi \in[\mu, \nu]$, we take $\xi=y+i b$, then $y \in[a, 0]$ and the second equation is

$$
\lim _{z \rightarrow t, t \in(\mu, v)} \frac{1}{2 \pi i} \int_{0}^{a} \rho(y+i b)\left[\frac{1}{y-(\bar{z}+i b)}-\frac{1}{y-(z-i b)}\right] \mathrm{d} y .
$$

When $z \in \Omega$ and $z \rightarrow t \in(\mu, v)$, we obtain $\bar{z}+i b \in \mathbb{C}^{+}$and $\rightarrow \bar{t}+i b \in(0, a)$. Hence, from (23), the second equation is true. In the same way, when $\xi \in[v, 0]$, suppose $\xi=i y$, then $y \in[b, 0]$ and the third limit equals

$$
\lim _{z \rightarrow t \in(\nu, 0)} \frac{1}{2 \pi i} \int_{0}^{b} \rho(i y)\left[\frac{1}{y-i \bar{z}}-\frac{1}{y+i z}\right] \mathrm{d} y=\rho(t) .
$$

The proof is completed. 
Define the following Pompeiu-type operator:

$$
\begin{aligned}
A_{\alpha}[f](z)= & -\frac{1}{\pi} \int_{\Omega}\left\{f(\zeta) \sum_{m, n}\left[q_{m, n}(\zeta, z)-\frac{q_{m, n}(\zeta, \alpha)+q_{m, n}(\zeta, \bar{\alpha})}{2}\right]\right. \\
& \left.-\overline{f(\zeta)} \sum_{m, n}\left[q_{m, n}(\bar{\zeta}, z)-\frac{q_{m, n}(\bar{\zeta}, \alpha)+q_{m, n}(\bar{\zeta}, \bar{\alpha})}{2}\right]\right\} \mathrm{d} \xi \mathrm{d} \eta
\end{aligned}
$$

for $f \in L_{p}(\Omega, \mathbb{C}), p>2$ with $q_{m, n}$ given by (4) and $\alpha \in \Omega$.

Lemma 3.2 Iff $\in L_{p}(\Omega, \mathbb{C})$, $p>2$, then $A_{\alpha}[f](z) \in C(\bar{\Omega}, \mathbb{C})$ and $\frac{\partial A_{\alpha}[f](z)}{\partial \bar{z}}=f(z)$ for $z \in \Omega$.

Proof From the classical Pompeiu-type operator in [14],

$$
T[f](z)=-\frac{1}{\pi} \int_{\Omega} \frac{f(\zeta)}{\zeta-z} \mathrm{~d} \xi \mathrm{d} \eta \in C(\bar{\Omega}, \mathbb{C})
$$

and $\frac{\partial T[f](z)}{\partial \bar{z}}=f(z)$ for $z \in \Omega$. When $z \in \Omega$, then $z+\omega_{m n} \notin \Omega$ for $(m, n) \neq(0,0)$ and $\pm \bar{z}+$ $\omega_{m n},-z+\omega_{m n} \notin \Omega$. Thus by (4) and (24), we have $A_{\alpha}[f](z) \in C(\bar{\Omega}, \mathbb{C})$ and the integral in (24) is analytic for $z \in \Omega$ except for one term $T[f](z)$, therefore,

$$
\frac{\partial A_{\alpha}[f](z)}{\partial \bar{z}}=\frac{\partial\left(-\frac{1}{\pi} \int_{\Omega} \frac{f(\zeta)}{\zeta-z} \mathrm{~d} \xi \mathrm{d} \eta\right)}{\partial \bar{z}}=f(z)
$$

Lemma 3.3 For $f \in L_{p}(\Omega, \mathbb{C}), p>2$,

$$
\lim _{z \in \Omega, z \rightarrow t} \operatorname{Re} A_{\alpha}[f](z)=0, \quad t \in \partial \Omega
$$

Proof By (24), we obtain

$$
\operatorname{Re} A_{\alpha}[f](z)=-\frac{1}{2 \pi} \int_{\Omega}\{f(\zeta)[P(\zeta, z)+\Phi(\zeta, \alpha)]-\overline{f(\zeta)}[P(\bar{\zeta}, z)+\Phi(\bar{\zeta}, \alpha)]\} \mathrm{d} \xi \mathrm{d} \eta
$$

with

$$
P(\zeta, z)=\sum_{m, n}\left[q_{m, n}(\zeta, z)-q_{m,-n}(\zeta, \bar{z})\right]
$$

and

$$
\Phi(\zeta, z)=\frac{1}{2} \sum_{m, n}\left[q_{m,-n}(\zeta, \bar{z})-q_{m, n}(\zeta, \bar{z})+q_{m,-n}(\zeta, z)-q_{m, n}(\zeta, z)\right]
$$

Obviously, we know $\Phi(\zeta, \alpha)=\Phi(\bar{\zeta}, \alpha)=0$ for $\zeta \in \Omega$. Therefore,

$$
\begin{aligned}
\operatorname{Re} A_{\alpha}[f](z)= & -\frac{1}{2 \pi} \int_{\Omega}\left\{f(\zeta) \sum_{m, n}\left[q_{m, n}(\zeta, z)-q_{m,-n}(\zeta, \bar{z})\right]\right. \\
& \left.-\overline{f(\zeta)} \sum_{m, n}\left[q_{m, n}(\bar{\zeta}, z)-q_{m,-n}(\bar{\zeta}, \bar{z})\right]\right\} \mathrm{d} \xi \mathrm{d} \eta
\end{aligned}
$$


Furthermore, when $z \in \partial \Omega$, it satisfies equation (17) by replacing $\zeta$ with $z$. For $\zeta \in \Omega$, $z \in[\mu, \nu]$, we have $\bar{z}=z-2 b i$, then

$$
\sum_{m, n}\left[q_{m, n}(\zeta, z)-q_{m,-n}(\zeta, \bar{z})\right]=\sum_{m, n}\left[q_{m, n}(\zeta, z)-q_{m,-n}(\zeta, z-2 b i)\right]=0 .
$$

Similarly,

$$
\sum_{m, n}\left[q_{m, n}(\bar{\zeta}, z)-q_{m,-n}(\bar{\zeta}, \bar{z})\right]=0, \quad z \in[\mu, \nu], \zeta \in \Omega
$$

Also, for $\zeta \in \Omega$ and $z \in \partial \Omega \backslash[\mu, \nu]$,

$$
\sum_{m, n}\left[q_{m, n}(\zeta, z)-q_{m,-n}(\zeta, \bar{z})\right]=\sum_{m, n}\left[q_{m, n}(\bar{\zeta}, z)-q_{m,-n}(\bar{\zeta}, \bar{z})\right]=0,
$$

thus the proof is completed.

Consider the Schwarz-type operator

$$
S_{\alpha}[\gamma](z)=\frac{1}{\pi i} \int_{\partial \Omega} \gamma(\zeta) \sum_{m, n}\left[q_{m, n}(\zeta, z)-\frac{q_{m, n}(\zeta, \alpha)+q_{m, n}(\zeta, \bar{\alpha})}{2}\right] \mathrm{d} \zeta,
$$

where $q_{m, n}$ is given by $(4)$ and $\gamma \in C(\partial \Omega, \mathbb{R})$. Then we have

$$
\begin{aligned}
\operatorname{Re} S_{\alpha}[\gamma](z)= & \frac{1}{2 \pi i} \int_{\partial \Omega} \gamma(\zeta)\left\{\sum_{m, n}\left[q_{m, n}(\zeta, z)-\frac{q_{m, n}(\zeta, \alpha)+q_{m, n}(\zeta, \bar{\alpha})}{2}\right] \mathrm{d} \zeta\right. \\
& \left.-\sum_{m, n}\left[\overline{q_{m, n}(\zeta, z)}-\frac{\overline{q_{m, n}(\zeta, \alpha)}+\overline{q_{m, n}(\zeta, \bar{\alpha})}}{2}\right] \mathrm{d} \bar{\zeta}\right\}
\end{aligned}
$$

By (18),

$$
\begin{aligned}
\operatorname{Re} S_{\alpha}[\gamma](z)= & \frac{1}{2 \pi i} \int_{\partial \Omega} \gamma(\zeta)\left\{\sum_{m, n}\left[q_{m, n}(\zeta, z)-g_{m, n}(\zeta, \bar{z})\right]\right. \\
& \left.-\frac{1}{2} \sum_{m, n}\left[q_{m, n}(\zeta, \alpha)-g_{m, n}(\zeta, \bar{\alpha})+q_{m, n}(\zeta, \bar{\alpha})-g_{m, n}(\zeta, \alpha)\right]\right\} \mathrm{d} \zeta
\end{aligned}
$$

where $g_{m, n}$ is given by (16). From (16) and Lemma 2.1, for $\zeta \in \partial \Omega$,

$$
\sum_{m, n}\left[q_{m, n}(\zeta, \alpha)-g_{m, n}(\zeta, \bar{\alpha})+q_{m, n}(\zeta, \bar{\alpha})-g_{m, n}(\zeta, \alpha)\right]=0
$$

and

$$
\sum_{m, n}\left[q_{m, n}(\zeta, z)-g_{m, n}(\zeta, \bar{z})\right]=\sum_{m, n}\left[q_{m, n}(\zeta, z)-q_{m, n}(\zeta, \bar{z})\right],
$$

therefore,

$$
\operatorname{Re} S_{\alpha}[\gamma](z)=\frac{1}{2 \pi i} \int_{\partial \Omega} \gamma(\zeta) \sum_{m, n}\left[q_{m, n}(\zeta, z)-q_{m, n}(\zeta, \bar{z})\right] \mathrm{d} \zeta
$$


Lemma 3.4 For $\gamma \in C(\partial \Omega, \mathbb{R}), S_{\alpha}[\gamma](z)$ is analytic in $\Omega$, i.e.,

$$
\frac{\partial S_{\alpha}[\gamma](z)}{\partial \bar{z}}=0
$$

Proof From (25), the sum in integrand can be rewritten as

$$
\frac{1}{2} \sum_{m, n}\left\{\left[q_{m, n}(\zeta, z)-q_{m, n}(\zeta, \alpha)\right]+\left[q_{m, n}(\zeta, z)-q_{m, n}(\zeta, \bar{\alpha})\right]\right\}
$$

which is convergent for $\zeta \in \partial \Omega, z \in \Omega$ by Lemma 2.1. Obviously, from the expression of $q_{m, n}$ in (4), the integrand in $S_{\alpha}[\gamma](z)$ is analytic for $z \in \Omega$, hence the proof is completed.

Lemma 3.5 For $z \in \Omega, \gamma \in C(\partial \Omega, \mathbb{R})$,

$$
\lim _{z \rightarrow t} \frac{1}{2 \pi i} \int_{L}\left[\gamma(\zeta)-\gamma\left(t_{0}\right)\right] \sum_{m, n}\left[q_{m, n}(\zeta, z)-q_{m, n}(\zeta, \bar{z})\right] \mathrm{d} \zeta=\gamma(t)-\gamma\left(t_{0}\right),
$$

where $t \in L^{0} \cup\left\{t_{0}\right\}, L^{0}$ is L except for two endpoints and

$$
L= \begin{cases}{[\mu, v],} & \text { for } t_{0}=\mu \text { or } v \\ {[\nu, 0],} & \text { for } t_{0}=0 \text { or } v \\ {[0, a],} & \text { for } t_{0}=0 \text { or } a, \\ {[a, \mu],} & \text { for } t_{0}=a \text { or } \mu .\end{cases}
$$

Furthermore, for $t \in \partial \Omega \backslash L^{0}$,

$$
\lim _{z \rightarrow t} \frac{1}{2 \pi i} \int_{L}[\gamma(\zeta)-\gamma(t)] \sum_{m, n}\left[q_{m, n}(\zeta, z)-q_{m, n}(\zeta, \bar{z})\right] \mathrm{d} \zeta=0
$$

Proof When $L=[\mu, \nu], t_{0}=v$ and $t \in L^{0} \cup\left\{t_{0}\right\}=(\mu, \nu]$,

$$
\begin{aligned}
& \lim _{z \rightarrow t} \frac{1}{2 \pi i} \int_{[\mu, \nu]}[\gamma(\zeta)-\gamma(v)] \sum_{m, n}\left[q_{m, n}(\zeta, z)-q_{m, n}(\zeta, \bar{z})\right] \mathrm{d} \zeta \\
& \quad=\lim _{z \rightarrow t} \frac{1}{2 \pi i} \int_{[\mu, \nu]}[\gamma(\zeta)-\gamma(v)]\left[\frac{1}{\zeta-z}-\frac{1}{\zeta-\bar{z}-2 b i}+\frac{1}{\zeta+z-2 b i}-\frac{1}{\zeta+\bar{z}}\right] \mathrm{d} \zeta \\
& \quad=\lim _{z \rightarrow t} \frac{1}{2 \pi i} \int_{[\mu, v] \cup[v,-a+i b]} \Gamma(\zeta)\left[\frac{1}{\zeta-z}-\frac{1}{\zeta-\bar{z}-2 b i}\right] \mathrm{d} \zeta,
\end{aligned}
$$

where

$$
\Gamma(\zeta)= \begin{cases}\gamma(\zeta)-\gamma(v), & \zeta \in[\mu, v], \\ \gamma(v)-\gamma(2 b i-\zeta), & \zeta \in[v,-a+i b] .\end{cases}
$$

By $\Gamma \in C([\mu, v] \cup[v,-a+i b], \mathbb{R})$ and from the second equation in Lemma 3.1, the above limit is $\Gamma(t)=\gamma(t)-\gamma(v)$. That is,

$$
\lim _{z \rightarrow t} \frac{1}{2 \pi i} \int_{[\mu, \nu]}[\gamma(\zeta)-\gamma(\nu)] \sum_{m, n}\left[q_{m, n}(\zeta, z)-q_{m, n}(\zeta, \bar{z})\right] \mathrm{d} \zeta=\gamma(t)-\gamma(\nu), \quad t \in(\mu, \nu] .
$$


Similarly,

$$
\lim _{z \rightarrow t} \frac{1}{2 \pi i} \int_{[\mu, \nu]}[\gamma(\zeta)-\gamma(\mu)] \sum_{m, n}\left[q_{m, n}(\zeta, z)-q_{m, n}(\zeta, \bar{z})\right] \mathrm{d} \zeta=\gamma(t)-\gamma(\mu), \quad t \in[\mu, \nu) .
$$

In the same way, when $L$ and $t_{0}$ are in all other cases of (27), the result (27) is also true.

Furthermore, by (17), we obtain for $z \in[0, a] \cup[a, \mu) \cup(\nu, 0]$ and $\zeta \in[\mu, \nu], \sum_{m, n}\left[q_{m, n}(\zeta\right.$, $\left.z)-q_{m, n}(\zeta, \bar{z})\right]=0$, therefore, for $t \in[0, a] \cup[a, \mu) \cup(v, 0]$,

$$
\lim _{z \rightarrow t} \frac{1}{2 \pi i} \int_{[\mu, \nu]}[\gamma(\zeta)-\gamma(t)] \sum_{m, n}\left[q_{m, n}(\zeta, z)-q_{m, n}(\zeta, \bar{z})\right] \mathrm{d} \zeta=0
$$

Combining the result (27), we obtain (29) is also true for corner points $t=\mu, v$, hence (28) holds for $L=[\mu, v]$ and $t \in \partial \Omega \backslash(\mu, v)$. Similarly, (28) is also true for $L, t$ in the other cases. The proof is completed.

Lemma 3.6 For $\gamma \in C(\partial \Omega, \mathbb{R})$ and $t \in \partial \Omega$,

$$
\lim _{z \in \Omega, z \rightarrow t} \operatorname{Re} S_{\alpha}[\gamma](z)=\gamma(t)
$$

Proof From (26), we only need to prove

$$
\lim _{z \in \Omega, z \rightarrow t} \frac{1}{2 \pi i} \int_{\partial \Omega} \gamma(\zeta) \sum_{m, n}\left[q_{m, n}(\zeta, z)-q_{m, n}(\zeta, \bar{z})\right] \mathrm{d} \zeta=\gamma(t), \quad t \in \partial \Omega
$$

First of all, taking $w(z) \equiv 1$ and by (12), (13), we know

$$
\frac{1}{2 \pi i} \int_{\partial \Omega} Q_{M, N}(\zeta, z) \mathrm{d} \zeta=1, \quad z \in \Omega
$$

and

$$
\frac{1}{2 \pi i} \int_{\partial \Omega} Q_{M, N}(\zeta, \alpha) \mathrm{d} \zeta=1 .
$$

In particular, for $\alpha \in \Omega, \pm \bar{\alpha}+\omega_{m n}$ do not belong to $\Omega$, so we have

$$
\frac{1}{2 \pi i} \int_{\partial \Omega} Q_{M, N}(\zeta, \bar{\alpha}) \mathrm{d} \zeta=0
$$

Combining the above three equations and taking $(M, N) \rightarrow(\infty, \infty)$, thus

$$
S_{\alpha}[1](z)=\frac{1}{\pi i} \int_{\partial \Omega} \sum_{m, n}\left[q_{m, n}(\zeta, z)-\frac{q_{m, n}(\zeta, \alpha)+q_{m, n}(\zeta, \bar{\alpha})}{2}\right] \mathrm{d} \zeta=1
$$

Then from (26),

$$
\operatorname{Re} S_{\alpha}[1](z)=\frac{1}{2 \pi i} \int_{\partial \Omega} \sum_{m, n}\left[q_{m, n}(\zeta, z)-q_{m, n}(\zeta, \bar{z})\right] \mathrm{d} \zeta=1, \quad z \in \Omega
$$


hence,

$$
\begin{aligned}
& \lim _{z \in \Omega, z \rightarrow t} \operatorname{Re} S_{\alpha}[\gamma](z) \\
& =\lim _{z \in \Omega, z \rightarrow t} \frac{1}{2 \pi i} \int_{\partial \Omega}[\gamma(\zeta)-\gamma(t)] \sum_{m, n}\left[q_{m, n}(\zeta, z)-q_{m, n}(\zeta, \bar{z})\right] \mathrm{d} \zeta+\gamma(t) .
\end{aligned}
$$

When $t \in(\mu, \nu)$, we rewrite $\operatorname{Re} S_{\alpha}[\gamma](z)$ as

$$
\frac{1}{2 \pi i} \int_{\partial \Omega}[\gamma(\zeta)-\gamma(\mu)] \sum_{m, n}\left[q_{m, n}(\zeta, z)-q_{m, n}(\zeta, \bar{z})\right] \mathrm{d} \zeta+\gamma(\mu),
$$

thus from the results for $L=[\mu, v], t_{0}=\mu$ in (27) and for $L=[0, a],[a, \mu]$, and $[v, 0]$ in (28), we get $\lim _{z \in \Omega, z \rightarrow t} \operatorname{Re} S_{\alpha}[\gamma](z)=\gamma(t), t \in(\mu, v)$. Furthermore, by (32) and Lemma 3.5, (30) obviously holds for $t=\mu, v$. Similarly, when $t \in \partial \Omega \backslash[\mu, v]$, the result is also true. Then the proof is completed.

\section{Theorem 3.1 The Schwarz problem}

$$
\left\{\begin{array}{l}
\partial_{\bar{z}} w=f \quad \text { in } \Omega, \quad \operatorname{Re} w=\gamma \quad \text { on } \partial \Omega \\
\operatorname{Im} w(\alpha)=c, \quad c \in \mathbb{R}
\end{array}\right.
$$

for $f \in L_{p}(\Omega ; \mathbb{C}), p>2, \gamma \in C(\partial \Omega ; \mathbb{R})$, and $\alpha$ is a fixed point in $\Omega$, is uniquely solvable by

$$
w(z)=S_{\alpha}[\gamma](z)+A_{\alpha}[f](z)+i c,
$$

where $A_{\alpha}, S_{\alpha}$ are defined by (24) and (25), respectively.

Proof By Lemmas 3.2-3.4 and Lemma 3.6, $\varphi(z)=S_{\alpha}[\gamma](z)+A_{\alpha}[f](z)$ satisfies the first two conditions in (33). Suppose $\phi(z)=w(z)-\varphi(z)$, then $\phi(z)$ satisfies

$$
\left\{\begin{array}{l}
\partial_{\bar{z}} \phi=0 \quad \text { in } \Omega \\
\operatorname{Re} \phi=0 \quad \text { on } \partial \Omega
\end{array}\right.
$$

Then from Theorem 2.1, we know $\phi(z)=i \operatorname{Im} w(\alpha)=i c$, thus, the proof is completed.

Competing interests

The authors declare that they have no competing interests.

Authors' contributions

All authors contributed equally to the manuscript and typed, read, and approved the final manuscript.

\section{Author details}

${ }^{1}$ School of Statistics and Mathematics, Zhongnan University of Economics and Law, Wuhan, 430072, P.R. China. ${ }^{2}$ College of Mathematics and Econometrics, Hunan University, Changsha, 410082, P.R. China.

\section{Acknowledgements}

This paper is supported by the Fundamental Research Funds for the Central Universities. The authors would like to thank the referees for their valuable suggestion and comments. 
References

1. Abdymanapov, SA, Begehr, H, Tungatarov, AB: Some Schwarz problems in a quarter plane. Eurasian Math. J. 3, 22-35 (2005)

2. Akel, M, Hussein, H: Two basic boundary-value problems for the inhomogeneous Cauchy-Riemann equation in an infinite sector. Adv. Pure Appl. Math. 3, 315-328 (2012)

3. Begehr, H, Schmersau, D: The Schwarz problem for polyanalytic functions. Z. Anal. Anwend. 24, $341-351$ (2005)

4. Begehr, H, Gaertner, EA: Dirichlet problem for the inhomogeneous polyharmonic equation in the upper half plane. Georgian Math. J. 14(1), 33-52 (2007)

5. Begehr, H, Vaitekhovich, T: Schwarz problem in lens and lune. Complex Var. Elliptic Equ. 59(1), 76-84 (2014)

6. Begehr, H: Green function for a hyperbolic strip and a class of related plane domains. Appl. Anal. 93(11), 2370-2385 (2014)

7. Shupeyeva, B: Some basic boundary value problems for complex partial differential equations in quarter ring and half hexagon. Dissertation, FU, Berlin (2013). http://www.diss.fu-berlin.de/diss/receive/FUDISS-thesis-000000094596

8. Vaitekhovich, T: Boundary value problems for complex partial differential equations in a ring domain. Dissertation, FU, Berlin (2008). http://www.diss.fu-berlin.de/diss/receive/FUDISS-thesis-000000003859

9. Wang, Y, Wang, Y: Two boundary-value problems for the Cauchy-Riemann equation in a sector. Complex Anal. Oper. Theory 6, 1121-1138 (2012)

10. Wang, Y, Wang, Y: Schwarz-type problem of nonhomogeneous Cauchy-Riemann equation on a triangle. J. Math. Anal. Appl. 377, 557-570 (2011)

11. Begehr, H, Vaitekhovich, T: How to find harmonic Green functions in the plane. Complex Var. Elliptic Equ. 56(12), 1169-1181 (2011)

12. Begehr, H, Vaitekhovich, T: Green functions, reflections, and plane parqueting. Eurasian Math. J. 1, 17-31 (2010)

13. Begehr, H, Vaitekhovich, T: Harmonic Dirichlet problem for some equilateral triangle. Complex Var. Elliptic Equ. 57(2-4), 185-196 (2012)

14. Begehr, H: Complex Analytic Methods for Partial Differential Equations: An Introductory Text. World Scientific, Singapore (1994)

15. Begehr, H: Boundary value problems in complex analysis I. Bol. Asoc. Mat. Venez. XII, 65-85 (2005)

16. Begehr, H: Boundary value problems in complex analysis II. Bol. Asoc. Mat. Venez. XII, 217-250 (2005)

17. Stein, EM, Shakarchi, R: Complex Analysis. Princeton University Press, Princeton (2003)

\section{Submit your manuscript to a SpringerOpen ${ }^{\odot}$ journal and benefit from:}

- Convenient online submission

- Rigorous peer review

- Immediate publication on acceptance

Open access: articles freely available online

- High visibility within the field

- Retaining the copyright to your article 